\title{
The efficacy of diffusion weighted imaging and apparent diffusion coefficient mapping for meniscal tears in the knee
}

\author{
Volkan Kızılgöz, Hasan Aydın, Baki Hekimoğlu \\ Radiologist-Radiology Department, Dışkapı Y.B. Eğitim ve Araştırma Hastanesi İrfan Baştuğ Caddesi Altındağ, Ankara-Turkey \\ Email address: \\ volkankizilgoz@gmail.com(V. Kızılgöz),dr.hasanaydin@hotmail.com(H. Aydın), hekim@superonline.com(B. Hekimoğlu)
}

\section{To cite this article:}

Volkan Kızılgöz, Hasan Aydın, Baki Hekimoğlu. The Efficacy of Diffusion Weighted Imaging and Apparent Diffusion Coefficient Mapping for Meniscal Tears in the Knee. Science Journal of Clinical Medicine. Vol. 2, No. 6, 2013, pp. 171-175.

doi: $10.11648 /$ j.sjcm.20130206.15

\begin{abstract}
Objective : The aim of this study was to analyse the efficacy of Diffusion Weighted MR Imaging (DWI) and Apparent Diffusion Coefficient (ADC) mapping in the diagnosis of meniscal tears in the knee. Conclusion : DWI and ADC mapping technique are fast and easily applicable with routine MRI sequences and these new MRI techniques (for the knee) depicts very important information for menisci and cartilages of the knee.
\end{abstract}

Key words: Diffusion, Knee, Imaging, MRI, Weighted

\section{Introduction}

MR imaging (MRI) is a reliable method of evaluating the meniscal tears and injuries. MRI provides important datas about bone and soft tissue disorders in and around the knee joint. This imaging method is also useful to avoid unnecessary diagnostic arthroscopy procedures and guides for artroscopy before the surgery. T1 and PD-T2W SE (proton density - T2 weighted spin echo), FSE (fast spin echo), Gradient Echo (GE) sequences (with transverse, sagittal and coronal images) with or without Fat-Saturation (FS) are still used as a routine knee MR imaging protocol in radiology departments $(1,2)$. The accuracy of 3D-MR imaging for meniscal tears and other knee pathologies was shown to be more than $90 \%$ in several papers (1-4). New MRI sequences, using different physical principles of MR imaging, have been introduced lately to be an alternative of routine MRI sequences for different purposes. Herein the efficacy of 3D-DWI and ADC mapping with different bvalues is going to be analysed and the results will be compared with routine MRI sequences for meniscal tears.

\section{Basic Physical Principles of DWI and ADC Mapping}

Diffusion-weighted magnetic resonance imaging measures differences in water mobility within different tissue microstructures and characterizes tissues based on the random Brownian displacement of water molecules. In biological tissues, water molecules are restricted within tissue spesific barriers. Blood vessels, macromolecules and cell membranes provides restricted areas for water molecules in the tissues. There are three major compartments which water molecules displace from one compartment to another. These compartments are intracellular space, extracellular extravascular space and extracellular intravascular space. Extracellular extravascular space is more important than the mobility in intracellular space and displacement between the compartments in currently most used DWI sequence applications.

Diffusion studies are performed with field gradients in addition to the radiofrequency and gradient pulses used for conventional MRI. During the time of echo (TE), a pair of field gradients is used to perform "diffusion-encoding." Each gradient in this gradient pair will last a time $\delta$, with strength $G$ (usually in units of $\mathrm{mT} / \mathrm{m}$ ), and the pair is separated by a time (5). And the intensity of the signal will depend these parameters, by the formula;

$$
\mathrm{S}=\mathrm{S} 0 \exp (-\mathrm{b} \mathrm{ADC}) \text {, }
$$

where $\mathrm{ADC}$ is the apparent diffusion coefficient ( $\mathrm{ADC}$ is 
expressed in $\mathrm{mm}^{2} / \mathrm{s}$ ), $\mathrm{b}$ is the gradient factor (simply called the b-factor) and S0 is the signal intensity acquired when no diffusion gradients are used. The lasting time of gradient pair gradient strength $(\mathrm{G})$ and the time needed for gradient pair to seperate are the parameters that affect on the bfactor (5). The b-factor can be set by the applicator. The equation above suggests that signal intensity is reducted when diffusion weighted sequence is applied. The diffusing spins move inside the field gradient and each spin is affected differently by the field. This difference destroys the alignment of spins with each other. This misalignment or "dephasing" results as signal dropping (5). The motionprobing gradients provides sensitivity to water proton mobility when used with T2 weighted sequence. As the amount or speed of the proton movement increases the more signal loss is induced by these gradients. Besides, the signal loss will be higher with increasing $b$-values. The average mobility can be quantified as apparent diffusion coefficient (ADC) indicates the amount of signal loss with different b-values.

\section{General Information about the Menisci}

The menisci handles very important biomechenical functions in the knee since they can absorb shock and distribute load during dynamic loading. Also they assist in joint lubrication $(4,7,8)$. The menisci provide stability to the knee. In weight-bearing or non-weight-bearing body positions, (in extension or flexion) $50-90 \%$ of body weight is transmitted through the menisci $(4,7)$. They provide a nearly frictionless articulation that can perform extensive biomechanical maneuvers which minimize the stress effect on the joint by distributing the stress forces over a large area of articular cartilage and over the underlying bone, enhance the ability of articular cartilage $(7,8)$.

Meniscal tears increase stress on tibial and femoral articular cartilages, disable the load-bearing function of meniscus, ultimately leading to degeneration. Exaggerated meniscal tears may even cause spontaneous osteonecrosis (7-9).

MRI provides information that can be used to grade pathology, guide therapy, prognosticate conditions for a wide spectrum of orthopedic conditions in the knee. MR imaging has emerged as the primary imaging method for the knee since it is the most sensitive and also a noninvasive technique for the diagnosis of meniscal tears. MRI can be performed on high field or low field systems with a knee coil Use of a local coil is mandatory to maximize signal-to-noise ratio $(1,2,4,7,10)$. MR images are acquired in sagittal, coronal and transverse planes, with a field of view (FOV) of about $16 \mathrm{~cm}$, imaging matrix of about $192 * 256$, 3-4 mm slice thickness, an adequate signal-tonoise ratio and an appropriate receiver bandwidth $(1,2,7)$. Some authors reported that sensitivity of 3D-MR imaging with a standard knee protocol was $86-96 \%$ and specificity was $84-94 \%$ for diagnosing the medial meniscal tears and they reported $68-86 \%$ sensitivity and $92-98 \%$ specificity for the lateral meniscal tears $(1,2,11,12)$. With developing advances in MRI systems, technology and RF coils, new sequences are applicable both in the studies and in a daily practice nowadays.

\section{Using DWI and ADC Mapping in General Clinical Pratice}

The acquisition of diffusion weighted images and evaluation of the images with this sequence still lacks standardization, but this technique is rapidly developing and continuing to progress into our clinical practice. Measuring the diffusion properties of water within tissues, either unidirectional (isotropic) or multidirectional (anisotropic) is the basic principle to provide image contrast by DW imaging $(12,13)$. The majority of DW imaging studies has focused on the measurement of extracellular water diffusion (both the magnitude and direction of diffusion) in order to indicate free diffusion of water protons (13).

DWI and ADC mapping can be adversely affected by artifacts from motion (vascular pulsations, voluntary movements and respiration etc.) rather than from the diffusion of molecules. Motion artefacts can easily interfere with the quality of images $(12,13)$. There are some MR sequences as spin echo (SE), gradient echo (GE) and single-shot EPI (echoplanar imaging) which are used as a standard technique that can provide complete images fast (within a second) and which are robust to

motion and resistance to magnetic field inhomogeneities. DWI sensitivity to diffusion (as mentioned above, characterised by its b-value) can be adjusted by altering the combination of gradient pulse amplitude. The higher the bfactor, the more sensitive is the image to the effects of diffusion (12). ADC map is used to remove the effects different than diffusion (as T2 weighting). ADC mapping is created by combining two images (with and without DWI). ADC mapping can also be created by using two b-values (in order to create an ADC map free of all contrast influences other than the displacement of water during the application of the diffusion gradients) (12,13). ADC mapping with range of b-values from 0 to $1000 \mathrm{~s} / \mathrm{mm}^{2}$ can be applied. Besides ADC mapping has been shown to be more sensitive than DWI, as it is only influenced by the magnitude of the diffused water molecules (12).

This technique is often used for depiction of acute ischemia of brain but nowadays this sequence has been used for miscellaneous anatomic localizations and provides important datas either alone or combined with routine imaging sequences. Recently these MR imaging techniques have also been applied in the diagnosis of head and neck tumors, breast and prostate cancers, rectal carcinomas and the response of tumors against radiotherapy or chemotherapy(14-22). 


\section{Evaluation of Meniscal Tears}

An increased meniscal signal was regarded as a meniscal tear when it could communicate with the inferior, superior or free edge of meniscal surface (or more than one of those) on two consecutive images and planes (sagittal and coronal) in MR imaging $(2,23,24)$. Tears were categorised as horizontal, longitudunal, oblique, radial, and root tears. Horizontal tear is regarded as a tear parallel to the tibial plateau separating the meniscus into the upper and the lower parts; longitudinal tear is a vertical tear perpendicular to the tibial plateau extending parallelly to the main axis of the meniscus; oblique tear is a tear extending obliquely to the main axis of the meniscus; radial, a vertical tear that begins in the central free margin perpendicular both to the tibial plateau and the free edge of the meniscus; complex, multiple tears of more than one configuration and one cleavage orientation lacking continuity; root, defined as a tear in the posterior or anterior meniscal attachments $(4,23,25)$. The criterias for meniscal tears in DWI and ADC mapping were the same criterias as in routine MR imaging. All types of tears also classified as: medial meniscal tears and lateral meniscal tears in the study. The absence of meniscus or exaggerated tears causing great damage to meniscus tissue due to the tear was recorded as maceration. One of our cases had maceration in this study.

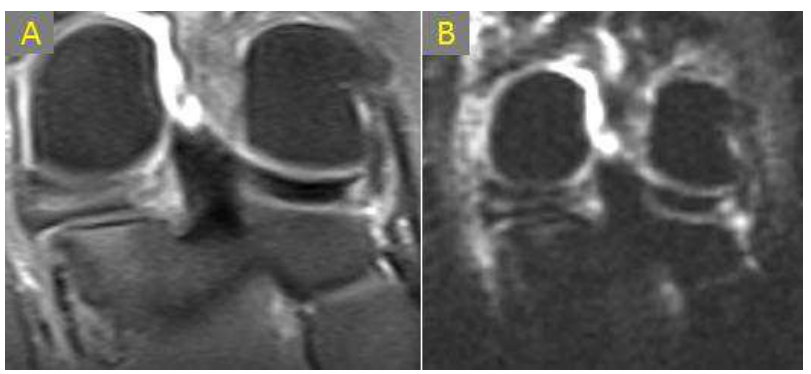

Figure 1. Medial meniscal tear presented with Proton Density Weighted (PDW) coronal fat saturated images (A) and DW image with b-value $50 \mathrm{~mm}^{2} / \mathrm{sec}$

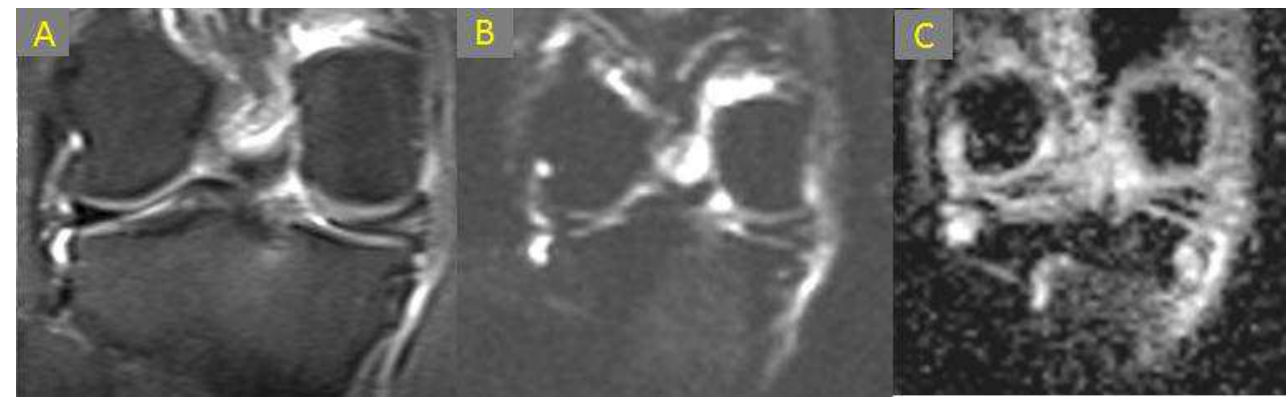

Figure 2. Medial meniscal tear shown by PDW (A), DW imaging with b-value $400 \mathrm{~mm}^{2} / \mathrm{sec}$ (B) and ADC mapping with b-value $400 \mathrm{sec} / \mathrm{mm}^{2}$ in coronal plane images.

Residual meniscal fragments after surgery and tears remaining after arthroscopy and were excluded from this study. Increased meniscal signal, located in the meniscal bodies, without any relationship to the inferior, superior or free edges of meniscal surfaces, was regarded as degeneration. Horizontal or triangular hyperintensity limited to meniscal bodies, was regarded as degenerated meniscal fragments. Oblique or vertical signals in articular surfaces and increased meniscal signals in meniscal attachment sites were never classified as degeneration. These criterias which indicated degeneration, were used for both routine and DWI-ADC mapping images.

\section{DWI-ADC Mapping in the Evaluation of Knee Joint, Including Menisci and Cartilage Injuries}

Aydin et al. reported a research about DWI and ADC mapping in the diagnosis of meniscal tears (26). He studied 74 consecutive( 30 men and 44 women) patients with meniscal tear under $1.5 \mathrm{~T}$ magnet, 11 of the patients had lateral meniscal tears, and 63 of them had medial meniscal tears which were consisted of 38 horizontal tears, 18 longitudinal tears, 2 radial tears, 2 root tears, and 7 complex and oblique tears. Lateral meniscal tears were of root, oblique and horizontal type, whereas medial meniscal tears included all types of tears. He had performed DWI by 3D-SE echo-planar imaging (EPI) in coronal and sagittal planes and ADC mapping in coronal planes with b-factors of 50,400 , and $800 \mathrm{sec} / \mathrm{mm}^{2}$.

He reported $100 \%$ specificity and $86 \%$ sensitivity with NPV of 0.09 and PPV of 1 for all meniscal tears according to the DWI results, regarded $100 \%$ specificity and $38 \%$ sensitivity by ADC mapping with b-value of $50 \mathrm{sec} / \mathrm{mm}^{2}$ with NPV of 0.02 and PPV of $1,100 \%$ specificity and $78 \%$ sensitivity with NPV of 0.06 and PPV of 1 by ADC mapping with b-value of $400 \mathrm{sec} / \mathrm{mm}^{2}$, and $100 \%$ specificity and $68 \%$ sensitivity with NPV of 0.04 and PPV of 1 for ADC mapping with b-value of $800 \mathrm{sec} / \mathrm{mm}^{2}$ with regard to routine MRI sequences.

In this research; ADC mapping with all applied b-values 
revealed no significant statistical differences when compared to routine MRI sequences, DWI and ADC mapping with b-value of $400 \mathrm{sec} / \mathrm{mm}^{2}$ revealed higher sensitivity and specificity than other imaging modalities(26).

As a result, he claimed that "DWI and ADC mapping, especially with b-factor of $400 \mathrm{sec} / \mathrm{mm} 2$, might be an alternative imaging modality to the routine MR imaging sequences for visualisation of meniscal tears especially for the medial meniscus. Despite their low spatial resolution, these scans were relatively fast and they should be easily used by an experienced musculoskeletal radiologist for detailed knee MR imaging".

Xu et al. (27) studied T2 mapping and diffusion weighted imaging in the diagnosis of early injury of knee cartilage under $3.0 \mathrm{~T}$ scanner. In this study, 72 subjects, including healthy group $(n=30)$ and early cartilage injury group $(n=42)$, were tested on MR scans with T2-mapping and DWI.

The normal group $(n=30)$ showed no abnormality in rutine MR imaging. 10 cases of 42 patients underwent conventional MR examination, in which the routine MR images depicted significant abnormal cartilages excluded from this study. 32 patients (the remaining patient group) with no cartilage abnormalities but different amounts of joint effusion were included in the study of early injury group. The result indicated that the average $\mathrm{T} 2$ values and ADC values of cartilage in early cartilage injury group were significantly higher than the normal group. The remarkable differences showed statistical significance.

\section{Conclusion}

There are not much studies mentioned about DWI and ADC mapping of knee in the literature so far. However, the studies in the literature about this subject indicates that these sequences are fast and easily applicable with routine MRI sequences and these new MRI techniques (for the knee) depicts very important information for menisci and cartilages of the knee, especially ADC mapping via b-value of $400 \mathrm{sec} / \mathrm{mm}^{2}$ for menisci of the knee moreover T2mapping and DW imaging are valuable imaging sequences in the diagnosis of early articular cartilage injury $(26,27)$.

\section{Author Contributions}

Guarantor of integrity of entire study : H.A,V.K.

Study design : V.K,H.A

Study concepts : V.K,H.A,B.H.

Data analysis : H.A.

Manuscript drafting : V.K

Literature research : H.A,V.K, B.H.

Clinical studies : H.A.

Statistical analysis : H.A.

Manuscript editing : V.K

Interpretation : H.A,V.K

\section{Financial Disclosure}

All authors state no financial relationship to disclose.

\section{References}

[1] Wolff AB, Pesce LL, Wu SJ et al: Comparison of spin echo T1W sequences versus fast spin-echo proton densityweighted sequences for evaluation of meniscal tears at $1.5 \mathrm{~T}$. Skeletal Radiol, 2009; 38(1): 21-29

[2] Ohishi T, Takahashi M, Abe $M$ et al: The use of axial reconstructed images from three-dimensional MRI datasets for morphological diagnosis of meniscal tears of the knee. Arch Orthop Trauma Surg, 2005; 125(2): 622-27

[3] Kocabey Y, Tetik O, Isbell WM et al: The value of clinical examination versus Magnetic resonance imaging in the diagnosis of meniscal tears and anterior cruciate ligament rupture. Arthroscopy, 2004; 20(7): 696-700

[4] Boxheimer L,Lutz AM, Zanetti M et al: Characteristics of displaceable and non-displaceable meniscal tears at kinematic MR imaging of the knee. Radiology, 2006; 238(1): 221-31

[5] Moritani T, Ekholm S, Westesson PL. Diffusion-Weighted MR Imaging of the Brain. Berlin: Springer-Verlag; 2004; 15 .

[6] Brown R (1928) A brief account of microscopical observations made in the months of June, July, and August, 1827 , on the particles contained in the pollen of plants; and on the general existence of active molecules in organic and inorganic bodies. Phil Mag 4:161-173

[7] Rubin DA, Palmer WE: Imaging of the knee. IDKD, 2005: 26-38

[8] Harper KW, Helms CA, Lambert HS et al: Radial meniscal tears: Significance, incidence and MR appearance. AJR, 2005; 185(4): 1429-34

[9] MR Quinn SF, Brown TR, Szumowski J: Menisci of the knee: Radial imaging correlated with arthroscopy in 259 patients. Radiology, 1992; 185(11): 577-80

[10] Rubin DA, Kneeland JB. MR imaging of the musculoskeletal system: technical considerations for enhancing image quality and diagnostic yield. AJR Am J Roentgenol 1994; 163:1155-1163

[11] Rubin DA, Paletta GA: Current concepts and controversies in meniscal imaging. Magn Reson Imaging Clin N Am, 2000; 8(3): 243-70

[12] Quinn SF, Brown TF: Meniscal tears diagnosed with MR imaging versus Arthroscopy: How reliable a standard is arthroscopy? Radiology, 1991; 181(12): 843-47

[13] Mori S, Barker BP: Diffusion magnetic resonance imaging: Its principle and applications. The anatomical record (New Anat), 1999; 257: 102-43

[14] Chawla S, Kim S, Dougherty L et al. Pretreatment diffusionweighted and dynamic contrast-enhanced MRI for prediction of local treatment response in squamous cell carcinomas of the head and neck. AJR Am J Roentgenol. 2013 Jan;200(1):35-43. 
[15] Nakajo M, Nakajo M, Kajiya $\mathrm{Y}$ et al. FDG PET/CT and diffusion-weighted imaging of head and neck squamous cell carcinoma: comparison of prognostic significance between primary tumor standardized uptake value and apparent diffusion coefficient. Clin Nucl Med. 2012 May;37(5):47580 .

[16] Verhappen MH, Pouwels PJ, Ljumanovic R. et al. Diffusionweighted MR imaging in head and neck cancer: comparison between half-fourier acquired single-shot turbo spin-echo and EPI techniques. AJNR Am J Neuroradiol. 2012 Aug;33(7):1239-46.

[17] Choi BB, Kim SH, Kang BJ. et al. Diffusion-weighted imaging and FDG PET/CT: predicting the prognoses with apparent diffusion coefficient values and maximum standardized uptake values in patients with invasive ductal carcinoma. World J Surg Oncol. 2012 Jun 28;10:126.

[18] Thomassin-Naggara I, De Bazelaire C, Chopier J. et al. Diffusion-weighted MR imaging of the breast: advantages and pitfalls. Eur J Radiol. 2013 Mar;82(3):435-43.

[19] Curvo-Semedo L, Lambregts DM, Maas M. et al. Diffusionweighted MRI in rectal cancer: apparent diffusion coefficient as a potential noninvasive marker of tumor aggressiveness. J Magn Reson Imaging. 2012 Jun;35(6):1365-71.

[20] Jang KM, Kim SH, Choi D et al. Pathological correlation with diffusion restriction on diffusion-weighted imaging in patients with pathological complete response after neoadjuvant chemoradiation therapy for locally advanced rectal cancer: preliminary results. $\mathrm{Br}$ J Radiol. 2012 Sep;85(1017):e566-72.
[21] Aydin H, Hekimoglu B, Kızılgöz V. A brief review of the combined use of T2-weighted MRI and diffusion-weighted imaging for prostate cancer diagnosis. AJR Am J Roentgenol. 2013 Feb;200(2):W219.

[22] Aydin H, Kizilgöz V, Tatar IG. et al. Detection of prostate cancer with magnetic resonance imaging: optimization of T1-weighted, T2-weighted, dynamic-enhanced T1-weighted, diffusion-weighted imaging apparent diffusion coefficient mapping sequences and MR spectroscopy, correlated with biopsy and histopathological findings. J Comput Assist Tomogr. 2012 Jan-Feb;36(1):30-45.

[23] Englund M, Guermazi A, Gale D et al: Incidental meniscal findings on knee MRI I middle-aged and elderly persons. N Engl J Med, 2008; 359(11): 1108-15

[24] Quinn SF, Brown TF: Meniscal tears diagnosed with MR imaging versus Arthroscopy: How reliable a standard is arthroscopy? Radiology, 1991; 181(12): 843-47

[25] Koenig JH, Ranawat AS, Umans HR et al: Meniscal root tears: Diagnosis and treatment. Arthroscopy, 2009; 25(9): $1025-32$

[26] Aydin H, Kızılgöz V, Hekimoğlu B. Is the quantitative Diffusion-Weighted MR Imaging and ADC mapping with bvalues of 50,400 , and $800 \mathrm{sec} / \mathrm{mm} 2$ a reliable method for evaluation of meniscal tears in the knee? Pol J Radiol, 2011; 76(1): 30-40

[27] Value of T2-mapping and DWI in the diagnosis of early knee cartilage injury. Jinfa Xu, Guohua Xie, Yujin Di et al. Radiology Case. 2011 Feb; 5(2):13-18 